\title{
Chidamide suppresses the glycolysis of triple negative breast cancer cells partially by targeting the miR-33a-5p-LDHA axis
}

\author{
XIANGDONG BAI ${ }^{1}$, HONGCHUAN JIANG ${ }^{2}$, GUOHUI HAN $^{1}$ and QIANG HE $^{3}$ \\ ${ }^{1}$ Department of Breast Surgery, Shanxi Provincial Cancer Hospital, Shanxi Medical University, Taiyuan, Shanxi 030013; \\ Departments of ${ }^{2}$ Breast Surgery and ${ }^{3}$ Hepatobiliary Surgery, Beijing Chaoyang Hospital, \\ The Affiliated Hospital of Capital Medical University, Beijing 100020, P.R. China
}

Received December 11, 2018; Accepted June 4, 2019

DOI: $10.3892 / \mathrm{mmr} .2019 .10425$

\begin{abstract}
Triple negative breast cancer (TNBC) is one of the most aggressive types of breast cancer and has a poor prognosis. Therefore, the development of novel drugs and understanding the molecular mechanisms that may contribute to the initiation and development of TNBC are urgently required. Chidamide, a histone deacetylase inhibitor, has been reported as possessing anti-cancer properties in several cancers, however, the function of chidamide in TNBC remains to be elucidated. The present study revealed that chidamide inhibited the proliferation, colony formation and migration of TNBC cells. Experiments investigating the underlying mechanism revealed that chidamide upregulated the expression of microRNA (miR)-33a-5p in TNBC cells via RT-qPCR. Luciferase reporter assay demonstrated that miR-33a-5p was bound to the 3'-untranslated region of lactate dehydrogenase $A$ (LDHA) and decreased the expression of LDHA in TNBC cells. In addition, chidamide suppressed the expression of LDHA and significantly decreased the glycolysis of TNBC cells. Collectively, the results of the present study demonstrated that chidamide reprogramed glucose metabolism, partially by targeting the miR-33a-5p/LDHA pathway, in TNBC. These findings indicate that chidamide may be a promising novel drug in the treatment of patients with TNBC.
\end{abstract}

\section{Introduction}

Triple negative breast cancer (TNBC) is one of the most aggressive subtypes of breast cancer, which is characterized by the lack of estrogen receptor, progesterone receptor and human epidermal growth factor receptor 2 (1-4). Due to

Correspondence to: Dr Qiang He, Department of Hepatobiliary Surgery, Beijing Chaoyang Hospital, The Affiliated Hospital of Capital Medical University, 8 South Road, Worker's Stadium, Chaoyang, Beijing 100020, P.R. China

E-mail: heqiang349@126.com

Key words: microRNA-33a-5p, triple negative breast cancer, lactate dehydrogenase A, glycolysis these clinical features, the response of patients with TNBC to conventional therapies remains unsatisfactory. Therefore, identifying novel drugs that may be applied in the treatment of TNBC and furthering our understanding of the underlying molecular mechanisms are urgently required.

Aberrant gene expression contributes substantially to the initiation and development of cancer in humans. It is well documented that epigenetic alternations regulate gene expression (5). In particular, the acetylation of histone proteins, which is catalyzed by histone acetyltransferases and histone deacetylases (HDACs), is the most extensively investigated epigenetic modification (6,7). HDACs have been revealed to be upregulated in a variety of human cancer types, promoting the progression of cancer (6-12). An increased expression of HDACs has been correlated with a poorer prognosis in patients with cancer. Previous studies have also demonstrated that disrupting the expression of HDACs with an HDAC inhibitor (HDACi) markedly suppressed the progression of cancer (13-16). These HDACis included quisinostat, entinostat and chidamide (17-22). It has been reported that chidamide is an inhibitor of class I HDACs, with specificity in targeting HDAC 1, 2, 3 and 10. The anticancer effects of chidamide have been reported in pancreatic cancer, non-small cell lung cancer, colon cancer and NK/T lymphoma cells (19-23). However, the function of chidamide and the underlying molecular mechanisms by which chidamide regulates the proliferation of TNBC cells remain to be fully elucidated.

MicroRNAs (miRNAs/miRs) are a class of small, non-coding RNAs with a length of 18-24 nucleotides, which modulate gene expression by binding to the 3'-untranslated region (3'-UTR) of downstream targets (24-26). The interactions between miRNAs and the 3'-UTR of target genes result in the degradation or translational inhibition of mRNAs (25). The aberrant expression of miRNAs is involved in the progression of human diseases. A recent study showed that renal miR-214-3p serves a functional role in the development of hypertension (27). miR-192-5p in the kidney protects against the development of hypertension (28). An increasing body of evidence demonstrates that miRNAs serve important roles in regulating the development of cancer by acting as tumor suppressors or oncogenes $(29,30)$. The aberrant expression of miRNAs confers resistance to drug treatment, therefore, modulating the expression of miRNAs has become a critical 
target of drugs in order to suppress the growth of cancer cells. As a specific characteristic of cancer, cancer cells metabolize glucose via aerobic glycolysis rather than mitochondrial aerobic respiration, even in conditions with sufficient oxygen $(31,32)$. The process of glycolysis is sequentially catalyzed by several enzymes, including glucose transporters, glucose-6-phosphate dehydrogenase and lactate dehydrogenase A (LDHA) (33). To regulate the growth of cancer cells, miRNAs have been found to modulate the expression of enzymes associated with glycolysis, which in turn regulates the metabolism of cancer cells. For example, miR-142-3p was shown to inhibit the aerobic glycolysis of hepatocellular carcinoma by targeting LDHA (34). Additionally, miR-30a-5p was reported to suppress the LDHA-mediated glucose metabolism of breast cancer cells (35). These studies indicate that miRNAs target LDHA and modulate the progression of cancer.

miR-33a-5p has been reported to be downregulated in melanoma, osteosarcoma and hepatocellular carcinoma, acting as a tumor suppressor in regulating the growth of cancer cells $(36,37)$. miR-33a-5p has been shown to increase the radiosensitivity of melanoma cells by inhibiting glycolysis (37). However, the expression and function of miR-33a-5p in TNBC remain unknown. In the present study, the results revealed that chidamide treatment suppressed the proliferation of TNBC cells. Further molecular investigations revealed that chidamide upregulated the expression of miR-33a-5p, which targeted LDHA and suppressed the glucose metabolism of TNBC cells. The results also demonstrated the possible functional mechanism of chidamide in inhibiting the growth of TNBC, which may be considered as a promising drug in the treatment of patients with TNBC.

\section{Materials and methods}

Clinical cancer tissues and cell lines. A total of 20 paired TNBC tissues and adjacent normal tissues were collected from patients with TNBC who had undergone surgical resection at Beijing Chaoyang Hospital between April 2013 and August 2014 (age: 29-66 years old; all patients were female and diagnosed with TNBC). The tissue samples were confirmed by three pathologists independently. None of these patients had received treatment prior to surgery. Written informed consent was obtained from all of participants prior to tissue collection. The samples were maintained at $-80^{\circ} \mathrm{C}$ until subsequent use. The Ethics Committee of The Affiliated Hospital of Capital Medical University (Beijing, China) approved the present study.

The MDA-MB-231 and BT-20 TNBC cell lines were purchased from American Type Culture Collection. The cells were cultured in RPMI-1640 medium containing $10 \%$ fetal bovine serum (FBS; Thermo Fisher Scientific, Inc.) and were maintained at $37^{\circ} \mathrm{C}$ in a humidified atmosphere with $5 \% \mathrm{CO}_{2}$. For transfection, $20 \mu \mathrm{M}$ miRNA was transfected into the TNBC cells using Lipofectamine 2000 (Invitrogen; Thermo Fisher Scientific, Inc.).

Cell proliferation assay. The MDA-MB-231 and BT-20 cells were seeded into 96 -well plates with 2,000 cells per well in $100 \mu \mathrm{l}$ of medium. After $24 \mathrm{~h}$, the cells were treated with increasing concentrations $(0,5,20,40,80,160$ and $320 \mathrm{nM})$ of chidamide for 24,48 and $72 \mathrm{~h}$ at $37^{\circ} \mathrm{C}$, respectively. To measure cell proliferation under different concentrations of chidamide, $20 \mu \mathrm{l}$ of Cell Counting Kit (CCK)-8 reagent (Beyotime Institute of Biotechnology) was added to the cells and incubated at $37^{\circ} \mathrm{C}$ for $3 \mathrm{~h}$. The absorbance of each well was measured at $450 \mathrm{~nm}$ with a microplate reader.

In vitro colony formation. A total of 3,000 cells/well were cultured in a 6-well plate with RPMI-1640 medium and treated with or without $80 \mathrm{nM}$ chidamide at the indicated concentration. Following incubation for 10 days at $37^{\circ} \mathrm{C}$, the medium was discarded and the cells were washed twice with PBS. The colonies were first fixed with $4 \%$ of paraformaldehyde at room temperature for $15 \mathrm{~min}$ and then stained with $1 \%$ crystal violet for $10 \mathrm{~min}$ at room temperature. The colonies were washed with PBS and counted via light microscopy.

RNA extraction and reverse transcription-quantitative polymerase chain reaction ( $R T-q P C R)$ analysis. RNA extraction from the tissues or cells was performed using TRIzol reagent (Invitrogen; Thermo Fisher Scientific, Inc.). The concentration of RNA was evaluated using the NanoDrop 2000 instrument (Thermo Fisher Scientific, Inc.). The RNA was first reverse transcribed into cDNA using the first-strand cDNA synthesis kit (Takara Biotechnology Co., Ltd.) with following the conditions: $25^{\circ} \mathrm{C}$ for $5 \mathrm{~min} ; 42^{\circ} \mathrm{C}$ for $20 \mathrm{~min}$ and $95^{\circ} \mathrm{C}$ for $1 \mathrm{~min}$. qPCR of the miR-33a-5p was conducted with SYBR Super mix (Applied Biosystems; Thermo Fisher Scientific, Inc.) on the 7900 Fast Real-Time PCR system (Applied Biosystems; Thermo Fisher Scientific, Inc.). The expression of GAPDH was detected as the endogenous control. The thermocycling conditions were as follows: $95^{\circ} \mathrm{C}$ for $10 \mathrm{~min}$, followed by 40 cycles at $95^{\circ} \mathrm{C}$ for $15 \mathrm{sec}$ and $60^{\circ} \mathrm{C}$ for $1 \mathrm{~min}$.

Targets prediction. The targets of miR-33a-5p were predicted using the TargetScan database (http://www.targetscan. org/vert_72/). Input 'miR-33a-5p' in the 'Enter a microRNA name' box and the targets were displayed following submission.

Luciferase reporter assay. The TNBC cells (10,000 per well) were cultured in 24-well plates and co-transfected with $20 \mathrm{nM}$ miR-33a-5p mimics (5'-GUGCAUUGUAGUUGC AUUGCA) or mimic control miRNA (5'-UUUGUACUACAC AAAAGUACUG) (Guangzhou RiboBio Co., Ltd.) and $250 \mathrm{ng}$ luciferase reporter vector (Promega Corporation) containing the wild-type or mutant 3'-UTR of LDHA. Transfection was performed with Lipofectamine $2000^{\mathrm{TM}}$ (Thermo Fisher Scientific, Inc.) according to the manufacturer's instructions. Following transfection for $48 \mathrm{~h}$, the cells were harvested and the luciferase reporter activity was measured using a Dual-Luciferase Assay kit (Promega Corporation) and normalized to Renilla luciferase activity. The experiment was performed in triplicate.

Western blot analysis. The MDA-MB-231 and BT-20 cells were collected and lysed with RIPA lysis buffer containing the protease inhibitor PMSF (Beyotime Institute of Biotechnology). The protein concentration was determined using a BCA assay (Beyotime Institute of Biotechnology). A total of $20 \mu \mathrm{g}$ of protein was loaded into $15 \%$ SDS-PAGE and then transferred onto polyvinylidene fluoride membranes (EMD Millipore). 
A

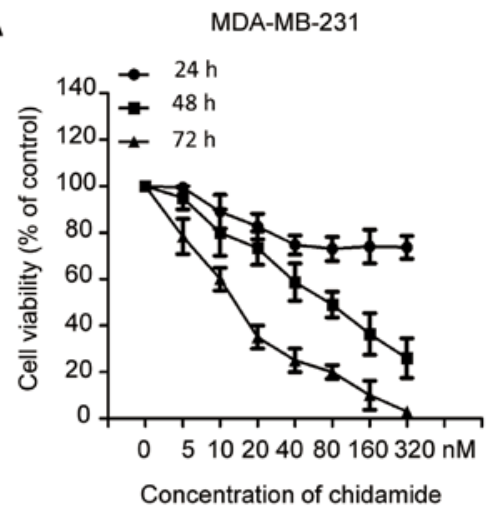

C

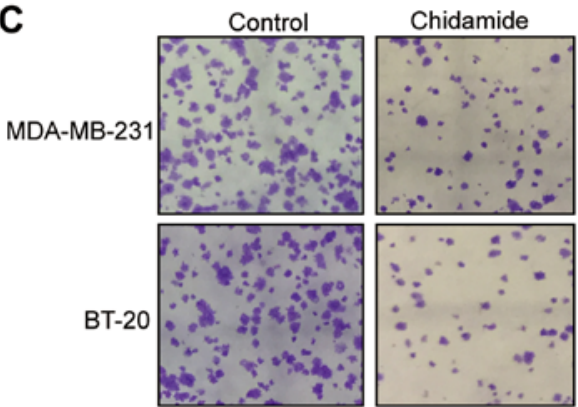

D
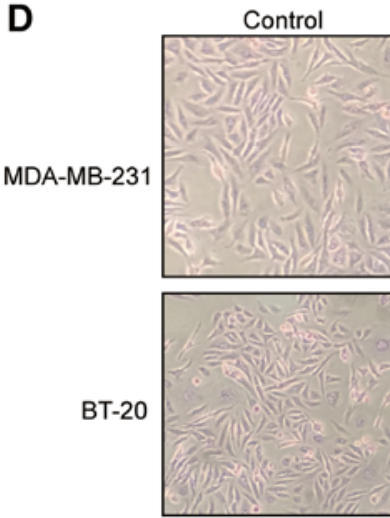

B

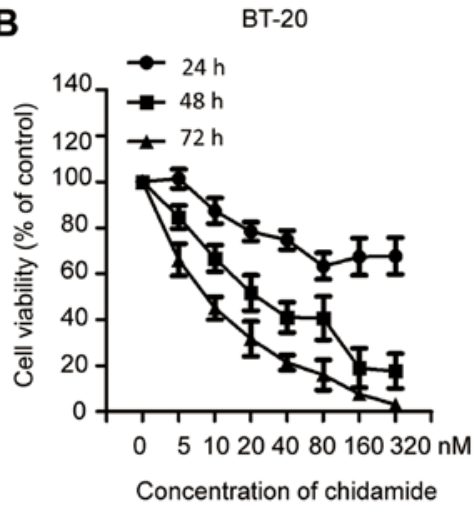

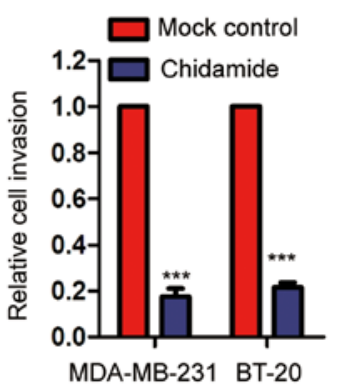

Figure 1. Chidamide suppresses the growth of TNBC cells. (A) MDA-MB-231 and (B) BT-20 cells were treated with increasing doses of chidamide for the 24 , 48 and $72 \mathrm{~h}$. Data were obtained from three independent experiments $(n=3)$. (C) Colony formation of TNBC cells with or without chidamide exposure $(n=3)$. Left-hand panel, representative image of the colonies (magnification, $\mathrm{x} 40$ ); right-hand panel, quantitative data of the colony numbers in each group. ${ }^{* * *} \mathrm{P}<0.001$, chidamide vs. Mock control. (D) Invasion of TNBC cells following treatment with chidamide was evaluated using a Transwell assay ( $\mathrm{n}=3$ ). Left-hand panel, representative image of the invasion of TNBC cells (magnification, $\mathrm{x} 40$ ); right-hand panel, quantitative data of the invasion numbers in each group. ${ }^{* * *} \mathrm{P}<0.001$, Chidamide vs. Mock control. TNBC, triple-negative breast cancer.

The membrane was first blocked with $5 \%$ non-fat milk at room temperature (RT) for $1 \mathrm{~h}$ and then incubated with the primary antibody overnight at $4^{\circ} \mathrm{C}$. Subsequently, the membrane was incubated with a secondary antibody conjugated to horseradish peroxidase (HRP) at RT for $1 \mathrm{~h}$ and the protein bands were visualized with an ECL detection kit (Thermo Fisher Scientific, Inc.) according to the manufacturer's instructions. The antibodies used in the present study included anti-LDHA (1:2,000 dilution; cat. no. 2012; Cell Signaling Technology, Inc.), anti-GAPDH (1:2,000 dilution; cat. no. 5174; Cell Signaling Technology, Inc.) and HRP-conjugated secondary antibody (1:10,000 dilution; cat. no. ZDR 5306; OriGene Technologies, Inc.).

Statistical analysis. The data are expressed as the mean \pm standard deviation. Statistical significance was analyzed using Student's t-test or one-way analysis of variance followed by Tukey's post hoc test. Statistical analyses were performed using SPSS 13.0 software (SPSS, Inc.). P $<0.05$ was considered to indicate a statistically significant difference.

\section{Results}

Chidamide treatment suppresses the growth of TNBC cells. To evaluate the effect of chidamide on the growth of TNBC cells, the MDA-MB-231 and BT-20 cells were treated with an increasing concentrations of chidamide for 24,48 and $72 \mathrm{~h}$, respectively. Cell proliferation was measured using a CCK-8 assay. As shown in Fig. 1A and B, chidamide significantly decreased the proliferation of MDA-MB-231 and BT-20 cells in a dose- and time-dependent manner. To further confirm these 
results, the influence of chidamide on the growth of TNBC cells was evaluated via an in vitro colony formation assay. The results indicated that chidamide treatment suppressed colony formation of the MDA-MB-231 and BT-20 cells (Fig. 1C). Additionally, the inhibitory effect of chidamide on the progression of TNBC cells was investigated using a cell invasion assay, which revealed that exposure to chidamide significantly attenuated the invasion of MDA-MB-231 and BT-20 cells (Fig. 1D). Collectively, these results demonstrated that chidamide treatment negatively regulated the growth of TNBC cells.

Chidamide upregulates the expression of $\mathrm{miR}-33 a-5 p$ in TNBC cells. Considering the significant suppressive effect of chidamide on the growth of TNBC cells, the possible underlying molecular mechanisms mediating the inhibitory function of chidamide were further investigated. An increasing body of evidence has suggested that miRNAs serve important roles in modulating drug sensitivity and cancer cell growth. To evaluate whether miRNAs are involved in chidamide-mediated cell growth inhibition in TNBC cells, our previous study screened the expression of miRNAs with MDA-MB-231 cells that were treated with chidamide. The data indicated that the expression level of miR-33a-5p was significantly increased the most with exposure to chidamide (Table SI). The present study confirmed this observation via the incubation of MDA-MB-231 and BT-20 cells with an increasing dose of chidamide, and the levels of miR-33a-5p were detected by RT-qPCR analysis. As presented in Fig. 2A, chidamide treatment significantly upregulated the expression of miR-33a-5p in a dose-dependent manner. To support this result, the expression of miR-33a-5p in TNBC cell lines and tissues was detected. The results revealed that the level of miR-33a-5p was decreased in the TNBC cell lines compared with that in the normal MCF-10A cells (Fig. 2B). Consistent with this, the expression of miR-33a-5p in TNBC tissues was significantly reduced compared with that in the paired adjacent normal tissues (Fig. 2C). The decreased expression of miR-33a-5p suggested the potential involvement of miR-33a-5p in the development of TNBC. To obtain further evidence, the MDA-MB-231 and BT-20 cells were transfected with miR-33a-5p mimics or control miRNA. The ectopic expression of miR-33a-5p was confirmed using an RT-qPCR assay (Fig. 2D). The proliferation of MDA-MB-231 and BT-20 cells was then detected using a CCK-8 assay. The results indicated that the overexpression of miR-33a-5p significantly decreased the proliferation rate of the TNBC cells (Fig. 2E and F). Consistently, transfection of the MDA-MB-231 and BT-20 cells with miR-33a-5p significantly inhibited colony formation and cell invasion (Fig. $2 \mathrm{G}$ and $\mathrm{H}$ ). To further confirm the regulation of miR-33a-5p on the growth of TNBC cells, the MDA-MB-231 and BT-20 cells were transfected with miR-33a-5p inhibitor to deplete the expression of miR-33a-5p (Fig. S1A). The CCK-8 assay revealed that the knockdown of miR-33a-5p significantly promoted the proliferation of TNBC cells (Fig. S1B and C). Consistently, the downregulation of miR-33a-5p enhanced the colony formation and invasion of the MDA-MB-231 and BT-20 cells (Fig. S1D and E). These results were suggestive of the tumor suppressive function of miR-33a-5p in the progression of TNBC.
miR-33a-5p targets LDHA in TNBC cells. To further understand the functional mechanisms by which miR-33a-5p modulated the growth of TNBC cells, the downstream targets of miR-33a-5p were predicted using the TargetScan database. Notably, LDHA was predicted as the putative downstream target of miR-33a-5p. The binding sites of miR-33a-5p at the 3'-UTR of LDHA are presented in Fig. 3A. To confirm the prediction, the MDA-MB-231 and BT-20 cells were co-transfected with miR-33a-5p mimics or control miRNA and a luciferase reporter vector containing the wild-type or mutant 3'-UTR of LDHA. The results revealed that transfection with miR-33a-5p significantly decreased the luciferase activity of the wild-type, but not the mutant, 3'-UTR of LDHA (Fig. 3B and C). To investigate whether the binding between miR-33a-5p with the 3'-UTR of LDHA affected the mRNA stability of LDHA, the MDA-MB-231 and BT-20 cells were transfected with miR-33a-5p mimics or control miRNA and the mRNA levels of LDHA were detected by RT-qPCR analysis. The results demonstrated that the overexpression of miR-33a-5p significantly decreased the mRNA expression of LDHA in the MDA-MB-231 and BT-20 cells (Fig. 3D). Additionally, to further characterize the importance of the binding between miR-33a-5p and the 3'-UTR of LDHA, nucleotides in miR-33a-5p were mutated. The results showed that mutated miR-33a-5p lost its ability to bind to the 3'-UTR of LDHA compared with the wild-type miR-33a-5p (Fig. S2A). Additionally, the expression of LDHA was unchanged by transfection with mutated miR-33a-5p in TNBC cells (Fig. S2B). To further characterize the negative regulation of miR-33a-5p on LDHA, the protein level of LDHA with ectopic expression of miR-33a-5p was also examined by western blotting. As shown in Fig. 3E, compared with the control group, a high expression of miR-33a-5p reduced the protein level of LDHA in the MDA-MB-231 and BT-20 cells. To further confirm the negative regulation of miR-33a-5p on the expression of LDHA, the expression of LDHA was detected in TNBC cells transfected with miR-33a-5p inhibitor. The results showed that the downregulation of miR-33a-5p increased the expression of LDHA at the mRNA and protein levels (Fig. 3F and G). These results demonstrated that miR-33a-5p bound the 3'-UTR of LDHA and suppressed the expression of LDHA in TNBC cells.

Chidamide reduces the glycolysis of TNBC cells. LDHA is an essential enzyme in the glycolysis of cancer cells. As overexpression suppressed the expression of LDHA in TNBC cells, to detect whether the aberrant expression of miR-33a-5p affected the glucose metabolism of TNBC cells, the MDA-MB-231 and BT-20 cells were transfected with miR-33a-5p mimics or control miRNA and glycolysis was evaluated. The results demonstrated that the ectopic expression of miR-33a-5p significantly decreased glucose uptake and lactate production in the MDA-MB-231 and BT-20 cells (Fig. 4A and B), which indicated that the overexpression of miR-33a-5p suppressed the glycolysis of TNBC cells.

As chidamide upregulated the expression of miR-33a-5p in TNBC cells, to further analyze the effect of chidamide on the glycolysis of TNBC cells, the MDA-MB-231 and BT-20 cells were treated with increasing concentrations of chidamide and the mRNA and protein levels of LDHA were examined by 
A

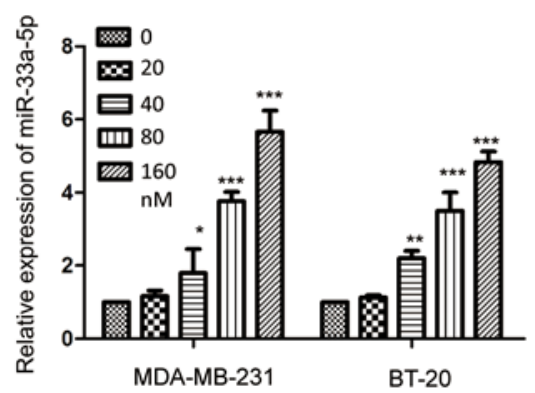

B

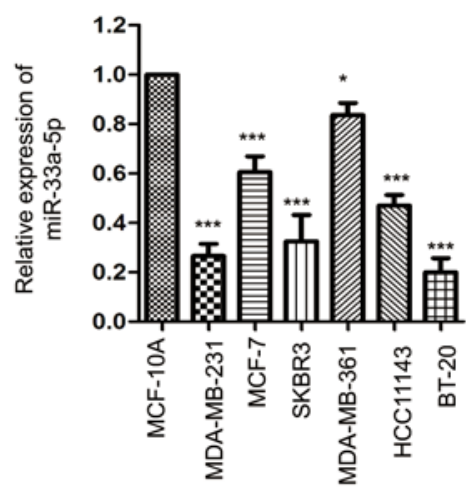

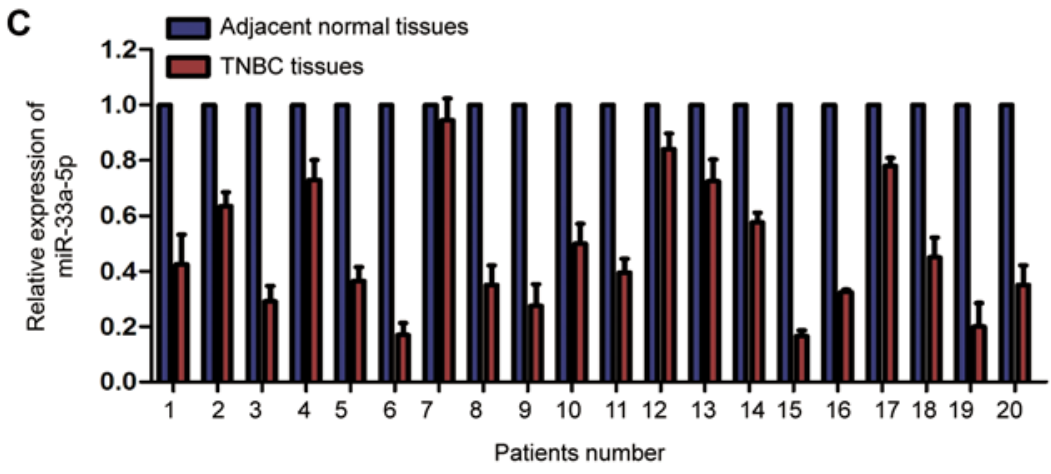

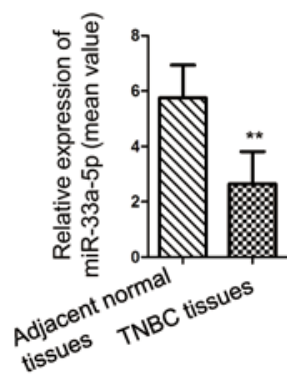

D

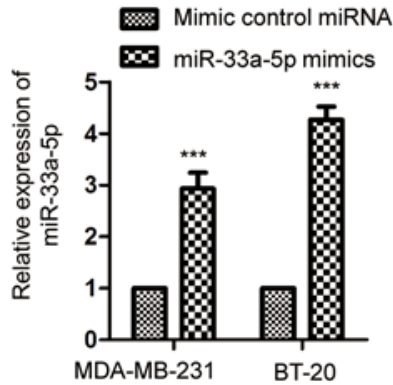

G

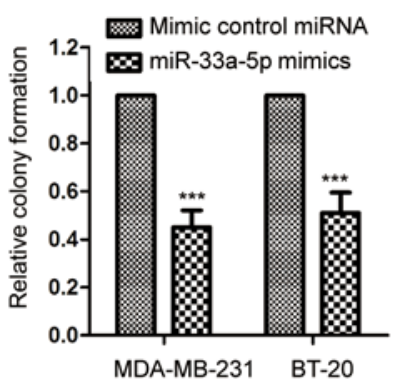

E MDA-MB-231
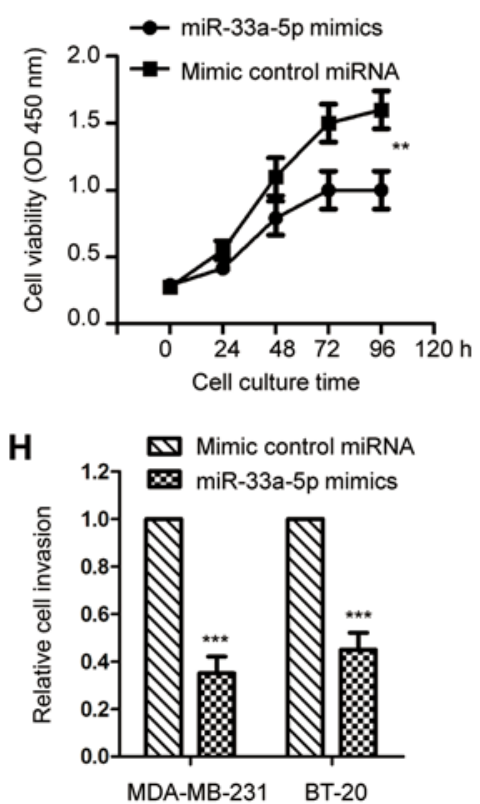

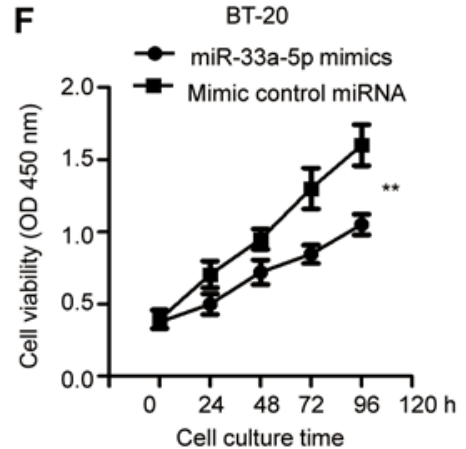

F

Figure 2. Chidamide upregulates the expression of miR-33a-5p in TNBC cells. (A) MDA-MB-231 and BT-20 cells were treated with increasing concentrations of chidamide for $48 \mathrm{~h}$ and the expression of miR-33a-5p was determined by RT-qPCR analysis $(\mathrm{n}=3)$. ${ }^{*} \mathrm{P}<0.05,{ }^{* * *} \mathrm{P}<0.01,{ }^{* * * *} \mathrm{P}<0.001$, chidamide $(20,40,80$ and $160 \mathrm{nM}$ ) vs. Mock control (0 nM). (B) Expression levels of miR-33a-5p in normal breast cells (MCF-10A) and different breast cancer cell lines were compared $(\mathrm{n}=3) .{ }^{*} \mathrm{P}<0.05$; ${ }^{* * *} \mathrm{P}<0.001$, compared with the expression of miR-33a-5p in MCF-10A. (C) Level of miR-33a-5p in 20 paired TNBC tissues and adjacent normal tissues were detected by RT-qPCR analysis. The mean values for the expression of miR-33a-5p in TNBC tissue and normal tissues are shown in the right-hand panel. "* $\mathrm{P}<0.01 \mathrm{TNBC}$ tissues vs. Adjacent normal tissues. (D) MDA-MB-231 and BT-20 cells were transfected with miR-33a-5p mimics or control miRNA and the ectopic expression of miR-33a-5p was determined via RT-qPCR analysis $(n=3)$. ${ }^{* * *} \mathrm{P}<0.001$, miR-33a-5p mimics vs. control miRNA group. Proliferation of (E) MDA-MB-231 and (F) BT-20 cells transfected with miR-33a-5p or control miRNA was detected using a Cell Counting Kit-8 assay (n=3). ${ }^{* *} \mathrm{P}<0.01$ miR-33a-5p mimics vs. control miRNA group. Overexpression of miR-33a-5p inhibited $(\mathrm{G})$ colony formation and $(\mathrm{H})$ cell invasion $(\mathrm{n}=3) .{ }^{* * * *} \mathrm{P}<0.001$ miR-33a-5p mimics vs. control miRNA group. TNBC, triple-negative breast cancer; RT-qPCR, reverse transcription-quantitative polymerase chain reaction; miR, microRNA.

RT-qPCR analysis and western blotting, respectively. The results revealed that exposure to chidamide decreased the levels of LDHA in these two cell lines (Fig. 4C and D). The present study also measured glucose consumption and lactate generation by treating MDA-MB-231 and BT-20 cells with chidamide. The results suggested that chidamide significantly reduced glucose 
A

Position of 654-661 of LDHA 3'-UTR

hsa-miR-33a-5p

B

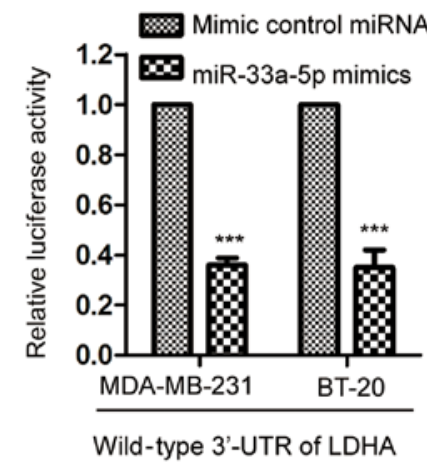

D

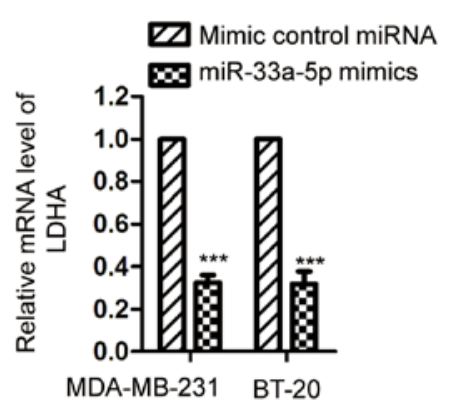

$\mathbf{F}$

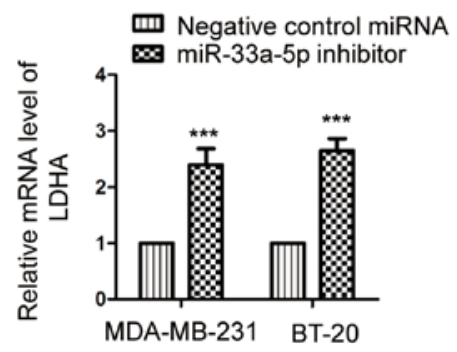

5'-AGAUCUACAUACAAACAAUGCAA

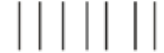

3'-ACGUUACGUUGAUGUUACGUG

C

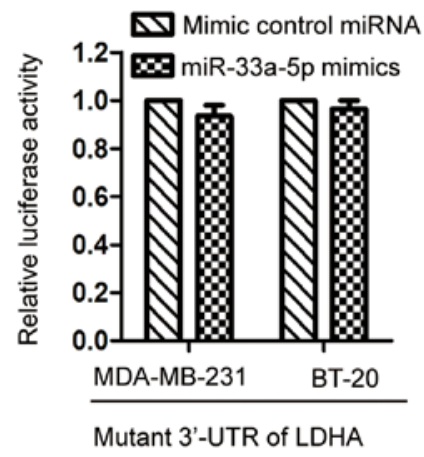

E Mimic control miR-33a-5p

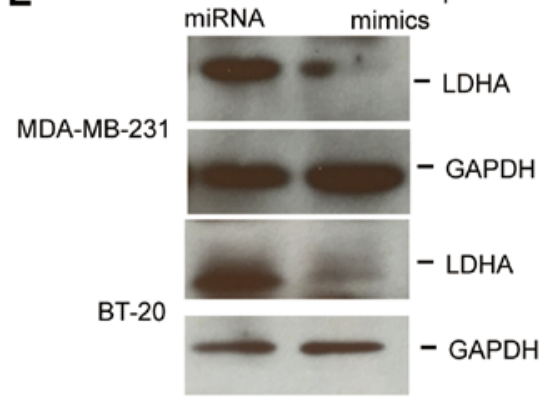

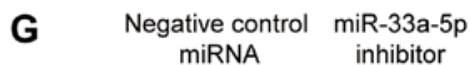

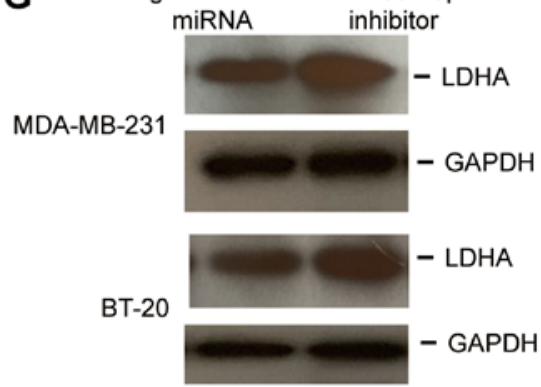

Figure 3. miR-33a-5p targets LDHA in TNBC cells. (A) Putative binding sites of miR-33a-5p at the 3'-UTR of LDHA were predicted. Luciferase reporter assay with MDA-MB-231 or BT-20 cells that were administered the (B) wild-type or (C) mutant 3'-UTR of LDHA in the presence of miR-33a-5p mimics $(\mathrm{n}=3) .{ }^{* * *} \mathrm{P}<0.001$, miR-33a-5p mimics vs. control miRNA. (D) mRNAs level of LDHA in TNBC cells expressing control miRNA or miR-33a-5p mimics were determined by RT-qPCR analysis $(\mathrm{n}=3) .{ }^{* * *} \mathrm{P}<0.001$, miR-33a-5p mimics vs. control miRNA. (E) Protein levels of LDHA in MDA-MB-231 and BT-20 cells harboring control miRNA or miR-33a-5p were evaluated by western blot analysis with anti-LDHA antibody $(\mathrm{n}=3$ ). The expression of GAPDH was also detected as the loading control. MDA-MB-231 and BT-20 cells were transfected with negative control miRNA or miR-33a-5p inhibitor, and the (F) mRNA and (G) protein levels of LDHA were detected $(\mathrm{n}=3){ }^{*}{ }^{* * *} \mathrm{P}<0.001$ miR-33a-5p inhibitor vs. negative control miRNA. TNBC, triple-negative breast cancer; RT-qPCR, reverse transcription-quantitative polymerase chain reaction; miR/miRNA, microRNA; LDHA, lactate dehydrogenase A; UTR, untranslated region.

uptake and lactate production in TNBC cells (Fig. 4E and F). These results demonstrated that chidamide upregulated miR-33a-5p, which consequently decreased the expression of LDHA and resulted in defects in the glycolysis of TNBC cells.

\section{Discussion}

Patients with TNBC respond poorly to the currently available chemotherapeutic strategies, which indicates the need for novel, efficient treatments to improve the outcomes for these patients. Due to the critical involvement of epigenetic modifications in modulating gene expression and cell growth, targeting enzymes that mediate epigenetic changes have been reported to suppress the progression of cancer. The promising anticancer effect of HDAC has emerged in a recent study (38). In the present study, exposure to chidamide significantly decreased the proliferation of TNBC cells by modulating the glycolysis of TNBC cells, which highlights the potential application of chidamide in the treatment of TNBC.

Chidamide has been identified as a class I HDACi, which was generated and has been approved for use in clinical practice in China $(39,40)$. Previous studies revealed that chidamide treatment led to defects in the growth of a variety 
A

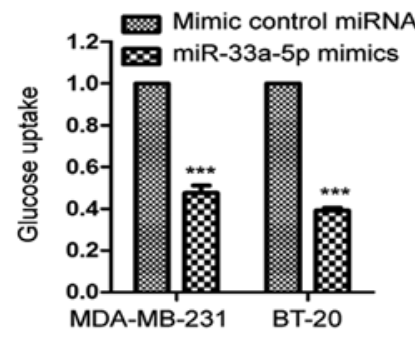

D

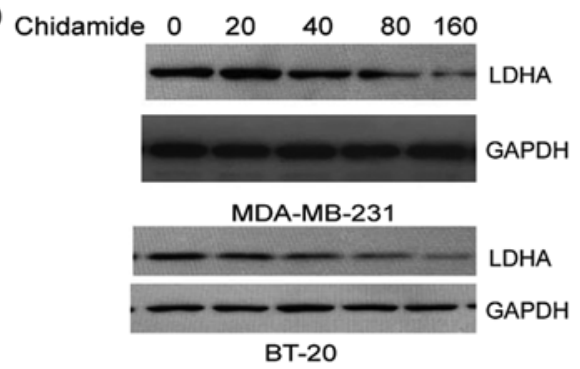

B

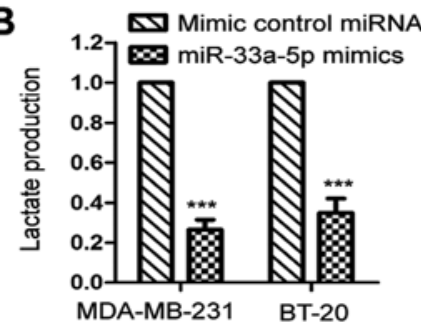

E

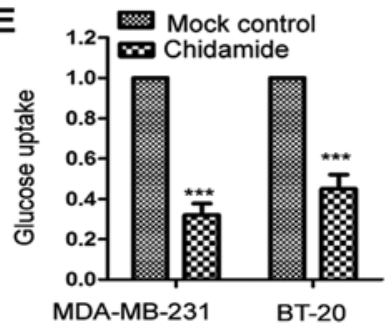

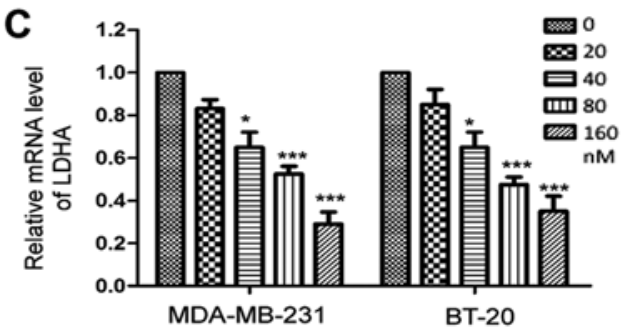

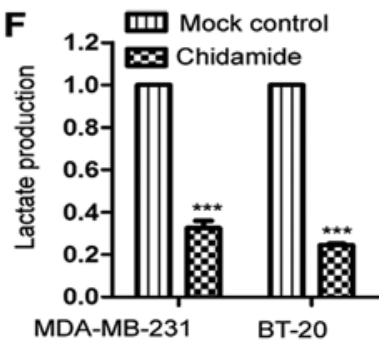

Figure 4. Chidamide inhibits the glycolysis of TNBC cells. MDA-MB-231 and BT-20 cells were transfected with miR-33a-5p mimics or control miRNA, and the (A) glucose uptake and (B) lactate production were measured $(\mathrm{n}=3)$. ${ }^{* * *} \mathrm{P}<0.001 \mathrm{miR}-33 \mathrm{a}-5 \mathrm{p}$ mimics vs. control miRNA. The TNBC cells were treated with an increasing dose of chidamide and the $(C)$ mRNA and (D) protein levels of LDHA were detected $(n=3) .{ }^{*} \mathrm{P}<0.05,{ }^{* * *} \mathrm{P}<0.001$, chidamide $(20,40,80$ and $160 \mathrm{nM})$ vs. Mock control (0 nM). (E) Glucose consumption and (F) lactate generation of MDA-MB-231 and BT-20 cells exposed to chidamide were examined $(\mathrm{n}=3) .{ }^{* * * *} \mathrm{P}<0.001$, chidamide vs. Mock control. TNBC, triple-negative breast cancer; miR/miRNA, microRNA; LDHA, lactate dehydrogenase A.

of cancer cells. For example, chidamide reduced proliferation and induced apoptosis in NK/T lymphoma cells by regulating the serine/threonine kinase-checkpoint kinase 2-p53-p21 signaling pathway (23). The inhibitory effect of chidamide was also observed in blastic plasmacytoid dendritic cell neoplasia (41). However, to the best of our knowledge, the function of chidamide in breast cancer, particularly in TNBC, has not been illustrated. In the present study, MDA-MB-231 and BT-20 cells were treated with chidamide and the results revealed that chidamide suppressed the proliferation, colony formation and migration of the TNBC cells. Further in vivo experiments may elucidate the growth inhibitory role of chidamide in TNBC. Additionally, it would be of interest to examine whether chidamide also reduces the progression of non-TNBC breast cancer cells.

An increasing body of evidence has suggested the critical involvement of miRNAs in the development of human cancer $(42,43)$. In the present study, the results demonstrated that miR-33a-5p was upregulated following chidamide treatment in TNBC cells. Previous studies demonstrated that the expression of miR-33a-5p was decreased, and inhibited the proliferation of lung adenocarcinoma cells and osteosarcoma cells $(36,44)$. In the present study, the overexpression of miR-33a-5p inhibited the proliferation of MDA-MB-231 and BT-20 cells. Further molecular investigations revealed that miR-33a-5p targeted LDHA and suppressed the expression of LHDA in TNBC cells. Consistent with the upregulation of miR-33a-5p, exposure to chidamide significantly decreased the levels of LDHA, which consequently suppressed the glycolysis of TNBC cells. A previous study indicated the overexpression of LDHA in TNBC tissues (45). It is noteworthy that miR-33a-5p was also observed to inhibit the glycolysis of melanoma by targeting hypoxia-inducible factor- $1 \alpha$, and the defects in glycolysis caused by the overexpression of miR-33a-5p conferred radiosensitivity to melanoma cells (37).
These results demonstrate that miR-33a-5p is a novel regulator of glucose metabolism by modulating the expression of enzymes that are essential for the glycolysis of cancer cells. It will be useful to examine whether the negative regulation of miR-33a-5p and chidamide on glucose metabolism also occurs in other types of cancer. Additionally, other miRNAs that may be involved in the anticancer effect of chidamide warrant further investigation. There were several limitations of the present study that require further improvements. For example, most of the results were obtained from in vitro experiments, indicating the need for in vivo experiments to further validate the effects of chidamide on the growth of TNBC cells. It may also be necessary to investigate the involvement of miRNAs other than miR-33a-5p in the suppressive function of chidamide in the progression of TNBC. The complete molecular mechanism by which chidamide regulates the expression of miR-33a-5p in TNBC cells remains to be fully elucidated.

In conclusion, the results of the present study revealed that chidamide inhibited the proliferation of TNBC cells, likely by upregulating the expression of miR-33a-5p. A high expression of miR-33a-5p decreased the expression of LDHA and suppressed the glycolysis of TNBC cells. These data indicate a possible mechanism by which chidamide inhibited the growth of TNBS cells, which suggests the potential application of chidamide in the treatment of patients with TNBC.

\section{Acknowledgements}

Not applicable.

\section{Funding}

This study was supported by a research grant from China's National Natural Science Foundation (grant no. 81471590) and by Chipscreen Biosciences Co., Ltd. 


\section{Availability of data and materials}

The datasets used and/or analyzed during the current study are available from the corresponding author on reasonable request.

\section{Authors' contributions}

$\mathrm{XB}$ and $\mathrm{QH}$ designed the study. XB performed the experiments. HJ collected the tissues and performed the RT-qPCR. $\mathrm{GH}$ helped with the data analysis. $\mathrm{XB}$ and $\mathrm{QH}$ wrote the manuscript. All authors read and approved the final manuscript.

\section{Ethics approval and consent to participate}

Written informed consent was obtained from all of participants prior to tissue collection. The Ethics Committee of The Affiliated Hospital of Capital Medical University (Beijing, China) approved the present study.

\section{Patient consent for publication}

Not applicable.

\section{Competing interests}

The authors declare that they have no competing interests.

\section{References}

1. Kandula M, Ch KK and Ys AR: Molecular mechanism and targeted therapy options of triple-negative (ER, PgR, HER-2/neu) breast cancer: Review. World J Oncol 4: 137-141, 2013.

2. Jamdade VS, Sethi N, Mundhe NA, Kumar P, Lahkar M and Sinha N: Therapeutic targets of triple-negative breast cancer: A review. Br J Pharmacol 172: 4228-4237, 2015.

3. Zeichner SB, Terawaki $\mathrm{H}$ and Gogineni K: A Review of systemic treatment in metastatic triple-negative breast cancer. Breast Cancer (Auckl) 10: 25-36, 2016.

4. Grubb W, Young R, Efird J, Jindal C and Biswas T: Local therapy for triple-negative breast cancer: A comprehensive review. Future Oncol 13: 1721-1730, 2017.

5. Liu Z, Gao Y and Li X: Cancer epigenetics and the potential of epigenetic drugs for treating solid tumors. Exp Rev Anticancer Ther, 2018 (Epud ahead of print).

6. Perri F, Longo F, Giuliano M, Sabbatino F, Favia G, Ionna F, Addeo R, Della Vittoria Scarpati G. Di Lorenzo G and Pisconti S: Epigenetic control of gene expression: Potential implications for cancer treatment. Crit Rev Oncol Hematol 111: 166-172, 2017.

7. Haberland M, Montgomery RL and Olson EN: The many roles of histone deacetylases in development and physiology: Implications for disease and therapy. Nat Rev Genet 10: 32-42, 2009.

8. Mei S, Ho AD and Mahlknecht U: Role of histone deacetylase inhibitors in the treatment of cancer (Review). Int J Oncol 25: 1509-1519, 2004.

9. Bruserud O, Stapnes C, Ersvaer E, Gjertsen BT and Ryningen A: Histone deacetylase inhibitors in cancer treatment: A review of the clinical toxicity and the modulation of gene expression in cancer cell. Curr Pharm Biotechnol 8: 388-400, 2007.

10. Hrabeta J, Stiborova M, Adam V, Kizek R and Eckschlager T: Histone deacetylase inhibitors in cancer therapy. A review. Biomed Pap Med Fac Univ Palacky Olomouc Czech Repub 158: 161-169, 2014.

11. Valente S and Mai A: Small-molecule inhibitors of histone deacetylase for the treatment of cancer and non-cancer diseases: A patent review (2011-2013). Expert Opin Ther Pat 24: 401-415, 2014.

12. Ali SR, Humphreys KJ, McKinnon RA and Michael MZ: Impact of histone deacetylase inhibitors on microRNA expression and cancer therapy: A review. Drug Dev Res 76: 296-317, 2015.
13. Hiriyan J, Shivarudraiah P, Gavara G, Annamalai P, Natesan S, Sambasivam G and Sukumaran SK: Discovery of PAT-1102, a novel, potent and orally active histone deacetylase inhibitor with antitumor activity in cancer mouse models. Anticancer Res 35: 229-237, 2015.

14. Min A, Im SA, Kim DK, Song SH, Kim HJ, Lee KH, Kim TY, Han SW, Oh DY, Kim TY, et al: Histone deacetylase inhibitor, suberoylanilide hydroxamic acid (SAHA), enhances anti-tumor effects of the poly (ADP-ribose) polymerase (PARP) inhibitor olaparib in triple-negative breast cancer cells. Breast Cancer Res 17: 33, 2015.

15. Schech A, Kazi A, Yu S, Shah P and Sabnis G: Histone deacetylase inhibitor entinostat inhibits tumor-initiating cells in Triple-negative breast cancer Cells. Mol Cancer Ther 14: 1848-1857, 2015.

16. Yang L, Liang Q, Shen K, Ma L, An N, Deng W, Fei Z and Liu J: A novel class I histone deacetylase inhibitor, I-7ab, induces apoptosis and arrests cell cycle progression in human colorectal cancer cells. Biomed Pharmacother 71: 70-78, 2015.

17. Zhong L, Zhou S, Tong R, Shi J, Bai L, Zhu Y, Duan X, Liu W, Bao J, Su L and Peng Q: Preclinical assessment of histone deacetylase inhibitor quisinostat as a therapeutic agent against esophageal squamous cell carcinoma. Invest New Drugs: Aug 31, 2018 (Epub ahead of print). doi: 10.1007/s10637-018-0651-4.

18. Connolly RM, Rudek MA and Piekarz R: Entinostat: A promising treatment option for patients with advanced breast cancer. Future Oncol 13: 1137-1148, 2017.

19. Liu L, Chen B, Qin S, Li S, He X, Qiu S, Zhao W and Zhao H: A novel histone deacetylase inhibitor Chidamide induces apoptosis of human colon cancer cells. Biochem Biophys Res Commun 392: 190-195, 2010.

20. Qiao Z, Ren S, Li W, Wang X, He M, Guo Y, Sun L, He Y, Ge Y and Yu Q: Chidamide, a novel histone deacetylase inhibitor, synergistically enhances gemcitabine cytotoxicity in pancreatic cancer cells. Biochem Biophys Res Commun 434: 95-101, 2013.

21. Zhou Y, Pan DS, Shan S, Zhu JZ, Zhang K, Yue XP, Nie LP, Wan J, Lu XP, Zhang W and Ning ZQ: Non-toxic dose chidamide synergistically enhances platinum-induced DNA damage responses and apoptosis in non-small-cell lung cancer cells. Biomed Pharmacother 68: 483-491, 2014.

22. Zhao B and He T: Chidamide, a histone deacetylase inhibitor, functions as a tumor inhibitor by modulating the ratio of Bax/Bcl-2 and P21 in pancreatic cancer. Oncol Rep 33: 304-310, 2015.

23. Zhou J, Zhang C, Sui X, Cao S, Tang F, Sun S, Wang S and Chen B: Histone deacetylase inhibitor chidamide induces growth inhibition and apoptosis in NK/T lymphoma cells through ATM-Chk2-p53-p21 signalling pathway. Invest New Drugs 36: 571-580, 2018

24. Bartel DP: MicroRNAs: Genomics, biogenesis, mechanism, and function. Cell 116: 281-297, 2004.

25. Fabian MR, Sonenberg N and Filipowicz W: Regulation of mRNA translation and stability by microRNAs. Annu Rev Biochem 79: 351-379, 2010

26. Mohr AM and Mott JL: Overview of microRNA biology. Semin Liver Dis 35: 3-11, 2015.

27. Liu Y, Usa K, Wang F, Liu P, Geurts AM, Li J, Williams AM, Regner KR, Kong Y, Liu H, et al: MicroRNA-214-3p in the kidney contributes to the development of hypertension. J Am Soc Nephrol 29: 2518-2528, 2018.

28. Baker MA, Wang F, Liu Y, Kriegel AJ, Geurts AM, Usa K, Xue H, Wang D, Kong Y and Liang M: miR-192-5p in the kidney protects against the development of hypertension. Hypertension 73: 399-406, 2019.

29. Kwak PB, Iwasaki S and Tomari Y: The microRNA pathway and cancer. Cancer Sci 101: 2309-2315, 2010.

30. Farazi TA, Spitzer JI, Morozov P and Tuschl T: miRNAs in human cancer. J Pathol 223: 102-115, 2011.

31. Zheng J: Energy metabolism of cancer: Glycolysis versus oxidative phosphorylation (Review). Oncol Lett 4: 1151-1157, 2012.

32. Akram M: Mini-review on glycolysis and cancer. J Cancer Educ 28: 454-457, 2013.

33. Li XB, Gu JD and Zhou QH: Review of aerobic glycolysis and its key enzymes-new targets for lung cancer therapy. Thorac Cancer 6: 17-24, 2015.

34. Hua S, Liu C, Liu L and Wu D: miR-142-3p inhibits aerobic glycolysis and cell proliferation in hepatocellular carcinoma via targeting LDHA. Biochem Biophys Res Commun 496: 947-954, 2018. 
35. Li L, Kang L, Zhao W, Feng Y, Liu W, Wang T, Mai H, Huang J, Chen S, Liang Y, et al: miR-30a-5p suppresses breast tumor growth and metastasis through inhibition of LDHA-mediated Warburg effect. Cancer Lett 400: 89-98, 2017.

36. Zhang J, Wang D, Xiong J, Chen L and Huang J: MicroRNA-33a-5p suppresses growth of osteosarcoma cells and is downregulated in human osteosarcoma. Oncol Lett 10: 2135-2141, 2015.

37. Cao K, Li J, Chen J, Qian L, Wang A, Chen X, Xiong W, Tang J, Tang S, Chen Y, et al: microRNA-33a-5p increases radiosensitivity by inhibiting glycolysis in melanoma. Oncotarget 8 : 83660-83672, 2017.

38. Tsilimigras DI, Ntanasis-Stathopoulos I, Moris D, Spartalis E and Pawlik TM: Histone deacetylase inhibitors in hepatocellular carcinoma: A therapeutic perspective. Surg Oncol 27: 611-618, 2018.

39. Lu X, Ning Z, Li Z, Cao H and Wang X: Development of chidamide for peripheral T-cell lymphoma, the first orphan drug approved in China. Intractable Rare Dis Res 5: 185-191, 2016.

40. Shi Y, Jia B, Xu W, Li W, Liu T, Liu P, Zhao W, Zhang H, Sun X, Yang H, et al: Chidamide in relapsed or refractory peripheral T cell lymphoma: A multicenter real-world study in China. J Hematol Oncol 10: 69, 2017.
41. Wang S, Guo W, Wan X, Teng Y, Zhou X and Bai O: Exploring the effect of chidamide on blastic plasmacytoid dendritic cell neoplasm: A case report and literature review. Ther Clin Risk Manag 14: 47-51, 2018.

42. Gentilin E, Degli Uberti E and Zatelli MC: Strategies to use microRNAs as therapeutic targets. Best Prac Res Clin Endocrinol Metab 30: 629-639, 2016.

43. Hosseinahli N, Aghapour M, Duijf PHG and Baradaran B: Treating cancer with microRNA replacement therapy: A literature review. J Cell Physiol 233: 5574-5588, 2018.

44. Pan J, Zhou C, Zhao X, He J, Tian H, Shen W, Han Y, Chen J, Fang S, Meng X, et al: A two-miRNA signature (miR-33a-5p and $\mathrm{miR}-128-3 \mathrm{p}$ ) in whole blood as potential biomarker for early diagnosis of lung cancer. Sci Rep 8: 16699, 2018.

45. Huang X, Li X, Xie X, Ye F, Chen B, Song C, Tang H and Xie X: High expressions of LDHA and AMPK as prognostic biomarkers for breast cancer. Breast 30: 39-46, 2016. 